\title{
Treatment of Gastric Cancer Patients During COVID-19 Pandemic: The West is More Vulnerable
}

This article was published in the following Dove Press journal: Cancer Management and Research

\section{Wojciech Piotr Polkowski Katarzyna Sędłak Karol Rawicz-Pruszyński}

Department of Surgical Oncology, Medical University of Lublin, Lublin, Poland
Correspondence: Wojciech Piotr Polkowski

Department of Surgical Oncology, Medical University of Lublin,

Radziwiłłowska 13 St, Lublin 20-080, Poland

Tel +48 8I 53I 8I 25

Fax +48815318133

Email wojciech.polkowski@uml.edu.pl

\begin{abstract}
The outbreak of the COVID-19 is currently the biggest international concern. Treatment of gastric cancer (GC) patients in the pandemic era with high hospital burden and under severe oncological/surgical resource constraints should implicate a need for resource re-allocation with a new "pandemic" GC treatment algorithm. The neoadjuvant/perioperative (radio-)chemotherapy is applied in the majority of advanced GC cases with poor postoperative therapy compliance. In the East, radical surgery is frequently used in the first instance, with adjuvant chemotherapy reserved for patients with a high risk of recurrence. Moreover, the elderly population might be effectively treated by surgery alone, thus saving oncological resources for younger people who need a more aggressive approach. In this framework, prioritization is a key concept based on the severity of symptoms and the need for urgent (surgical) intervention. High-risk and marginally effective surgery should be replaced with definitive radio- and/or chemotherapy. The pandemic framework to provide optimal care for GC patients must be based on multidisciplinary decision-making and include all anti-cancer treatment options: surgery, systemic therapy, and radiotherapy. The priority and staffing dictate adherence to the new algorithm. We believe that these priorities may improve the delivery of care to all, including elderly GC patients.
\end{abstract}

Keywords: COVID-19, gastric cancer, multidisciplinary treatment

\section{Introduction}

The Global Cancer Statistics in 2018 (GLOBOCAN) indicate that gastric cancer (GC) remains significant cancer worldwide. Being responsible for over 1000000 new cases in 2018 and an estimated 783000 deaths (equating to 1 in every 12 deaths globally), GC is the fifth most frequently diagnosed cancer and the third leading cause of cancer death. Among men, it is the most commonly diagnosed cancer and the main cause of cancer death in several Western Asian countries. Incidence rates are markedly elevated in Eastern Asia and Eastern Europe. ${ }^{1}$ However, globally there has been a steady decrease in GC incidence. ${ }^{2}$ In some countries GC should reach the rare disease threshold by $2035 .^{3}$ On the contrary, increasing risks have been observed in younger generations. The predicted growing number of new cases highlights that GC remains a major challenge to public health on a global scale.

GC outcome is fundamentally different depending on the world region. In the West, the survival rate is among the poorest of all solid-organ tumors, predominantly due to the frequent presence of metastatic (stage IV) disease at initial 
presentation. An observational study from the Eastern Public Hospital showed an increase in gastrointestinal cancers hospitalization, with GC presenting the highest inhospital mortality rate. ${ }^{4}$ This might be a reflection of epidemiological changes in the 21 st century, implicating a need for resource re-allocation due to high hospital burden.

\section{COVID-19 Outbreak}

The Coronavirus Disease 2019 (COVID-19) caused by SARS-CoV-2, is a novel rapidly spreading the infectious disease of the biggest international concern. The COVID19 dashboard from the Center for Systems Science and Engineering at Johns Hopkins University has shown 2594 724 total confirmed cases and 179778 deaths (last updated 17 July 2020). ${ }^{5}$ The Global Cancer Observatory (GCO) is an interactive web-based platform presenting global cancer statistics to inform cancer control and cancer research, ${ }^{6}$ by the International Agency for Research on Cancer of the World Health Organization. The list of the estimated number of new GC cases and deaths in 2018 (GCO), as well as confirmed COVID-19 cases and deaths in countries with a high incidence of GC or COVID-19 is presented in Table 1.

CRITCON levels are based on DEFCON levels, which report nuclear war probability. CRITCON is designed as a tool for Intensive Care Units capacity under conditions of the pandemic. It was initially designed within the NW London Critical Care Network in 2009 in response to the H1N1 influenza pandemic and the potential threat of triage

Table I Estimated Number of GC and COVID-19 New Cases and Deaths in Selected Countries

\begin{tabular}{|l|l|l|l|l|}
\hline Country & \multicolumn{2}{|l|}{$\begin{array}{l}\text { Gastric Cancer } \\
\text { (2018) }\end{array}$} & \multicolumn{2}{l|}{ COVID-I9 (I7 July 2020) } \\
\cline { 2 - 5 } & $\begin{array}{l}\text { New } \\
\text { Cases }\end{array}$ & Deaths & $\begin{array}{l}\text { Confirmed } \\
\text { Cases }\end{array}$ & Deaths \\
\hline US & 26026 & 11438 & 3576 I54 & 138358 \\
Brazil & 20927 & 15796 & 2012 I5I & 76688 \\
Italy & 12803 & 9457 & 243736 & 35017 \\
Spain & 7684 & 5609 & 258855 & 28416 \\
France & 7726 & 5326 & 211102 & 30141 \\
UK & 6370 & 4484 & 294116 & 45204 \\
Germany & 14173 & 9480 & 201450 & 9087 \\
Poland & 6659 & 5779 & 39054 & 1605 \\
Iran & 11644 & 8965 & 267061 & 13608 \\
China & 456124 & 390182 & 85314 & 4644 \\
Japan & 115546 & 48535 & 23510 & 985 \\
Korea & 37266 & 7684 & 13672 & 293 \\
\hline
\end{tabular}

by a resource (critical care rationing). The principle behind CRITCON is to allow each unit to declare itself in simple terms compared to its normal capacity (CRITCON 0-4, corresponding to "business as usual", "normal winter", "unprecedented", "last resort", and "triage"). This enables units under unusual stress to be instantly visible and to trigger mutual aid.

COVID-19 is a highly contagious, and the most important morbidity factor is exposure to an infection source. ${ }^{7}$ Patients with cancer are susceptible to the infection, resulting in poor outcome. ${ }^{8}$ The median age of COVID-19 patients with cancer (63 years) was reported to be significantly higher than for those without cancer (49 years), suggesting that older age is associated with worse COVID-19 outcomes.

In the COVID-19 outbreak, the significant risk for a cancer patient is the inability to receive necessary medical services both in terms of hospital admission and standard medical care administration. ${ }^{9}$ Additionally, hospitalization increases the risk of infection. Consequently, some treatment modalities are being delayed. Enforced quarantine complicates hospital admission for repeat chemotherapy cycles or radiotherapy fractions and may cause cancer care discontinuity. When severe complications or emergencies occur in advanced cases, treatment unavailability is of possible concern. Severe adverse effects, including neutropenic fever among patients who receive chemotherapy, are more challenging to diagnose and might not be treated promptly, which might affect survival. ${ }^{9}$ Delay of cancer treatment could lead to tumor progression and poor outcome, therefore each clinical strategy should be risk-adjusted and consulted with the patient.

Since the outcome of the COVID-19 pandemic may be highly variable, the American College of Surgeons has organized decision-making into three phases that describe the acuity of the local COVID-19 status (COVID-19 Guidelines for Triage of Cancer Surgery Patients). ${ }^{10}$ For elective cases with a high likelihood of postoperative ICU or respirator utilization, it will be more imperative that the risk of delay to the individual patient is balanced against the immediate availability of these resources for patients with COVID-19.

A worldwide pandemic forces a change in the daily risk and benefit assessment expected from the use of immunosuppressive and potentially toxic chemo- and/or radiotherapy, as well as major surgery in patients with GC. Both diseases, GC and COVID-19, although completely different in nature, have a dramatic impact on the societies of 
both hemispheres. Their comparison is aimed to provide useful suggestions for surgeons and oncologists who are facing the major challenge in the treatment decisionmaking of patients with GC who may be at higher risk of developing a severe and deadly SARS-CoV-2 infection.

\section{Current Standard of GC Treatment}

National Comprehensive Cancer Network (NCCN) guidelines recommend accurate clinical staging, perioperative therapy, and complete lymphadenectomy for patients with stage II to III GC. However, national compliance remains low. Complete clinical staging is available for $66 \%$ of patients, $27 \%$ undergo surgery alone $(73 \%$ receive adjuvant therapy), and 53\% have complete lymphadenectomy. Compliance with guidelines is associated with improved outcomes (median OS 46 months). ${ }^{11}$ Since the NCCN guidelines for GC are one of the most authoritative evidencebased recommendations worldwide, they are respected in other regions with a high proportion of advanced GC. ${ }^{12}$

Early (T1a) GC with no risk of lymph node metastases can be treated by endoscopy alone (EMR, ESD). Gastrectomy is potentially curative. Since the majority of patients relapse following surgery alone, combined modality therapy is standard for advanced (stage IB-III) disease. In Europe, perioperative chemotherapy (FLOT) is a preferred pathway. ${ }^{13,14}$ For patients undergoing upfront surgery without administration of preoperative chemotherapy, postoperative adjuvant radio- and/or chemotherapy is recommended. In the US, the use of neoadjuvant radiochemotherapy has been increasing, primarily in lymph node involvement $(\mathrm{cN}+) .{ }^{15}$ Patients with metastatic GC generally receive only palliative chemotherapy, but a proportion of stage IV patients with good response to induction systemic therapy could be re-assessed to achieve an R0 resection. However, the extent of such an aggressive surgical approach (lymphadenectomy, metastasectomy, multi-visceral resection) remains a subject of debate, since it harbors the substantial risk of severe morbidity and ICU stay. This so-called conversion therapy may be used when liver or peritoneal metastases that had been unresectable or marginally resectable (for technical and/or oncological reasons) became controllable by chemotherapy. ${ }^{16}$

\section{East vs West}

The regional variation between the East (Asia) and the West in treatment paradigms for $\mathrm{GC}$ has been well described. ${ }^{17}$ Major differences have been identified in tumor biology, implementation of screening programs, the extent of surgical lymphadenectomy, and routine use of neoadjuvant versus adjuvant treatment strategies. Neoadjuvant chemotherapy and adjuvant chemoradiotherapy are employed in the US and Europe, while adjuvant chemotherapy is standard in Asia. Minimally invasive techniques, including both laparoscopic and robotic platforms, have been studied in both regions, with attention to safety, feasibility, and long-term oncologic outcomes. However, due to the predominance of early GC in Asia (Japan, Korea), the laparoscopic/robotic approach is more common in the East than in the West.

The most recent version of the European Society for Medical Oncology (ESMO) Clinical Practice Guidelines for the diagnosis, treatment and follow-up of GC was published in $2016 .{ }^{13}$ In 2017 it was decided to convene special guidelines to adapt the ESMO guidelines taking into account the ethnic differences associated with the treatment of metastatic GC in Asian countries (Japan, China, Korea, Malaysia, Singapore, and Taiwan). ${ }^{18}$

However, the main difference between the East and the West is the role of surgery in the multidisciplinary treatment of advanced GC. ${ }^{19}$ An upfront standardized radical surgery with D2 lymphadenectomy is conventional in Asian countries, with a minimally-invasive approach for early GC. Whereas in the Western hemisphere, more limited surgery in terms of lymphadenectomy (D1 plus = modified D2), is usually preceded by preoperative neoadjuvant radio- (US) and/or chemotherapy (Europe), with nearly $50 \%$ of patients not receiving any adjuvant therapy after resection. This adjuvant chemotherapy (S1) is the standard of care in the East. ${ }^{20}$

Mass screening programs implemented in some of the Far East countries in the 20th century facilitated prompt GC diagnosis with less than $30 \%$ of new cases diagnosed at advanced (III-IV) stages. On the other hand, in Western countries, more than $60 \%$ of new GC cases are diagnosed at the advanced stages. The REGATE registry enrolled nearly 10,000 patients with newly diagnosed GC at any stage of the disease from 22 countries of the Asia-Pacific region, Europe, the Indian subcontinent, Latin America and North Africa between 2004 and 2008. ${ }^{21,22}$ Stage I GC was more frequent in Asia-Pacific (39\%) versus other regions (6 $-18 \%$ ), whereas stage IV GC was more frequent outside Asia-Pacific. Surgery was planned for most patients, although $69 \%$ of patients received surgery as originally planned. Overall, 15\% of patients received no treatment (Asia-Pacific 8\%; Indian subcontinent 25\%). Combined modality treatment was used in one-third of patients. Curative surgery alone was used most frequently for stages 
$0-$ II and was employed often in Europe (55\%) and the Asia-Pacific (48\%). The Asia-Pacific and Indian subcontinent patients were more likely to have a distal subtotal gastrectomy and less likely to undergo total gastrectomy than patients in other regions. Minimally-invasive gastrectomy has been established as a commonly used method for the early GC in Korea and Japan. ${ }^{23}$

\section{Elderly}

Elderly GC patients are often subjects of multidisciplinary management decisions throughout the world. Mortality among US patients undergoing major gastrointestinal resections steadily increases with age. ${ }^{24}$ The so-called "critical age" (75 \pm 2 years) was suggested to standardize and improve outcomes by focusing on perioperative resources. Adequate staging ( $>15$ lymph nodes examined) is less common in older patients. However, when compliance is reached, they are less likely to have node-positive tumors. Regardless of age, adherence to the national guidelines is associated with improved survival and may alter multimodality management of GC in the elderly. ${ }^{25}$ Based on the Surveillance Epidemiology and End Results (SEER) databases, out of nearly 80,000 GC patients who were recommended for surgery (1973-2014), nearly $20 \%$ of cases did not undergo surgery. Poor compliance was related to age, race, unsatisfactory grading, advanced stage, civil and socioeconomic status, and earlier time of diagnosis. GC patients of poor surgical compliance demonstrated adverse survival, which was comparable to that of non-surgical patients. ${ }^{26}$ In Korea, stage I and II GC patients, aged 70-79 years had a significantly higher rate of cancer-specific mortality than younger patients $(<50$ years). However, age was not an independently associated factor for cancer-specific mortality in patients with stage III and IV GC. Although considered as an independent risk factor for GC-related mortality, the impact of age may differ depending on the stage of cancer. ${ }^{27}$

In the Dutch CRITICS trial, out of 788 randomized patients, $22 \%$ were older ( $\geq 70$ years) adults with resectable GC who received preoperative chemotherapy (3 cycles of EOX/ECX), followed by surgery, and postoperative radio and/or chemotherapy. Although they frequently $(77 \%)$ experienced severe toxicity, and thus received lower relative dose intensities during preoperative chemotherapy, equal proportion $(80 \%)$ of older patients underwent curative surgery as compared to younger ones. Postoperative complications and mortality were comparable in both age groups. However, $36 \%$ of older patients did not start adjuvant therapy. Moreover, severe toxicity for chemo-radiotherapy was reported in nearly half of patients undergoing postoperative systemic treatment. Therefore, perioperative treatment compliance, especially in the postoperative phase, was poorer in older adults when compared with younger. ${ }^{28}$ This may be one of the reasons why older age is associated with worse cancer-specific mortality in the perioperative therapy setting that is recommended in Europe.

Treatment patterns and outcomes of non-metastatic elderly GC patients were studied in Alberta, Canada (2007-2012). Out of predominantly male (69\%) patients, in the median age of 80 , only $13 \%$ received multimodality therapy. Over $35 \%$ of surgical patients had grade II or higher complications. Perioperative mortality was $10 \%$. Nearly $40 \%$ of the patients recurred at a median of 13 months after surgery. There was no significant difference in 5-year DFS or OS (39\% - 47\%) between patients treated with surgery alone or with multimodality treatment. ${ }^{29}$ Thus, even with surgery alone, selected elderly patients with advanced GC can obtain apparent prolonged survival.

\section{Proximal Localization}

Additional mortality-related factor among elderly GC patients may be the localization of the primary tumor. Although GC is often reported as a single entity, it can generally be classified into two localization categories: proximal (cardia) and non-cardia tumors, arising from more distal regions of the stomach. Rates of non-cardia gastric cancer have been steadily declining over the last one-half century in most populations. ${ }^{1}$ Cancers of the gastric cardia (arising at the esophagogastric junction) have epidemiological characteristics more similar to those of esophageal adenocarcinoma, and important risk factors include obesity and gastroesophageal reflux disease, with Barrett esophagus also supposed to increase the risk. ${ }^{30}$ The incidence of these proximal GC has been increasing, particularly in high-income countries. ${ }^{31}$ For several populations, like for men in the US, UK, and Australia, estimated cardia GC rates were comparable with or exceeded estimated noncardia GC rates. ${ }^{32}$ Tumor location at a proximal site is more common in Europe, Latin America, and North Africa (approximately 20\%) than in Asia-Pacific, where the antral location is predominant. ${ }^{21}$

Using the 1998-2006 SEER database, it was shown that $61 \%$ of GC surgery was performed in patients $\geq 65$ years, less than $30 \%$ received adequate lymphadenectomy or adjuvant radiation therapy. The adverse effect of older 
age on cancer-specific mortality was augmented in proximal GC. ${ }^{33}$

For junctional tumors, proximal gastrectomy has been accepted procedure both in the East (for early GC), as in the West very recently. ${ }^{34}$ Proximal gastrectomy is feasible and may be safely used in patients with advanced GC after neoadjuvant chemotherapy with acceptable survival. ${ }^{35}$

\section{Strategy for the Treatment of GC During the COVID-19 Pandemic}

To minimize the adverse effects of the pandemic, an urgent need for treatment strategy change has been anticipated in Chinese literature. ${ }^{36}$ Surgical intervention for benign tumors should be postponed. For invasive GC, multidisciplinary therapy is recommended, and non-surgical anti-tumor therapy should be selected with higher priority. Neoadjuvant therapy is highly recommended for GC at advanced stages that meet the indications of NCCN guidelines (stage $\geq \mathrm{T} 2$ ). GC with obstruction can be managed with gastric tube decompression or stent placement to relieve the symptoms. Naso-gastric feeding tube placement/percutaneous endoscopic gastrostomy could be adopted to ensure enteral nutrition supply. Transcatheter arterial embolization for hemostasis is a choice for gastrointestinal bleeding. However, in case of an acute, uncontrolled bleeding, obstruction, or alternative treatment measures fail, an emergency operation must still be performed in a designated isolation area.

Tertiary prevention measures must be adopted for all anesthetists with an additional face mask or goggles to prevent respiratory droplet transmission. Accurate disinfection of the operating room after surgery is necessary. The postoperative fever must be carefully differentiated between abdominal infection/inflammation and COVID19. Single-room isolation and related examinations should be performed according to the standard procedures. ${ }^{37}$

There is an only limited suggestion from the Western perspective on a new strategy for the treatment of GC during the COVID-19 pandemic. $^{38}$ The COVID-19 pandemic is changing the organization of healthcare and has a direct impact on multimodality treatment of GC, including surgery. Surgical oncology priorities have been modified by several hospitalized COVID-19 patients, limitations of ICU access and respiratory support, operation room (OR) supplies, and transfusion capabilities (decreased blood donation): emergency surgery remains a priority, while functional surgery is to be deferred. Laparoscopic surgery must follow strict rules to avoid exposure of healthcare professionals to added risk. Predefined morbidity and mortality rates should be compared with the oncological risk incurred by deferring surgery and/or the tumor doubling time. Strategies can be proposed based on this comparison.

Surgery for GC carries a moderate risk of complications, with major complications rates of $10-15 \%$, mostly respiratory and septic. ${ }^{39,40}$ Localized GC generally progresses quite slowly irrespectively of the histological subtype. The average progression time from localized to locally advanced or metastatic disease is $34-44$ months. The doubling time of a locally advanced GC is 6.2 months. ${ }^{41,42}$ Since these data come from Asian countries, they need to be interpreted with caution in other parts of the world.

Current strategies for neoadjuvant chemotherapy (FLOT) or chemo-radiotherapy, extended total gastrectomies must be deferred, with a discussion of how to pursue preoperative treatment if it ends during the pandemic (maintenance chemotherapy according to modes discussed in multidisciplinary team meetings and adapted case-by-case, taking into account the non-negligible risk of chemo-induced immunosuppression). For partial gastrectomy, given the lower risk of complications, surgery can be proposed to patients at low operative risk. ${ }^{38}$

In the UK, COVID-19 has significantly impacted the multidisciplinary treatment of GC. The common strategy included two priorities: emergency cases and deferment of surgery. ${ }^{43}$ Patients presenting with bleeding GC or gastric outlet obstruction not amenable to endoscopic/interventional radiology were considered for surgery. Minimally invasive surgery was not feasible during the early phase of the pandemic. Staging laparoscopy has been postponed for newly diagnosed patients until completion of neoadjuvant treatment. Patients requiring extended resection have been considered for alternative treatments. This triage of GC patients in a resource-limited environment during the COVID-19 pandemic, have enabled the safe performance of potentially curative gastrectomy in the optimal number of patients.

In the European epicenter of the COVID-19 pandemic (Lombardy in Italy), the adapted institutional recommendations have been proposed for the systemic treatment of patients with $\mathrm{GC}^{44}$. For perioperative chemotherapy of patients with locally advanced GC, the choice of multimodality treatment over surgery alone has been made after careful evaluation of each individual risk-benefit profile. First, microsatellite instability (MSI) testing has been performed for all patients to avoid chemotherapy in those 
with MSI-high, amenable for upfront R0 resection. Preoperative chemotherapy has been recommended only for patients with age $<70$ years and high-risk resectable GC. Oxaliplatin-based doublets were the treatment of choice for patients with node-positive disease and selected patients with cT3N0 tumors with adverse prognostic features (diffuse histologic type) or technical risks of incomplete resection (esophagogastric junction localization). More toxic but effective FLOT (docetaxel, oxaliplatin, and fluorouracil/leucovorin) triplet chemotherapy has been recommended only for cT4 tumors and in cases with a high risk of positive margins (R1). For patients with esophagogastric junction (type 1 and 2 Siewert tumors), the use of neoadjuvant chemoradiation has been discouraged. For postoperative chemotherapy, the choice between single-agent fluoropyrimidines (capecitabine) or CAPOX (capecitabine plus oxaliplatin) has been based on the pathologic stage and individual risk of relapse.

We propose a new treatment algorithm for patients with GC with the proviso that it is strictly pandemicrelated (Figure 1). It is based on insights from GC management in elderly patients, as it must bear the features of simple and effective treatment with a low risk of complications and mortality.

To minimize the burden of oncological service and adjust the management to limited anesthesiology staff and intensive care resources, the following provisions have been proposed:

- staging laparoscopy should be maintained, with informed consent to proceed to gastrectomy if the primary tumor is resectable

- especially in non-intestinal tumors ${ }^{45}$

- for early GC following non-radical endoscopic resection (R1), the definitive radical surgery may be postponed

- neoadjuvant (radio-)chemotherapy for resectable cases deferred

- upfront gastrectomy preferred if easy resectable with low risk of respiratory complications

○ major GC surgery, as recently defined by experts, ${ }^{46}$ only in referral centers with proven low morbidity/ mortality rates, performed by experienced surgeons or under their strict supervision ${ }^{47}$

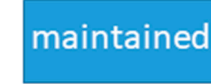

deferred

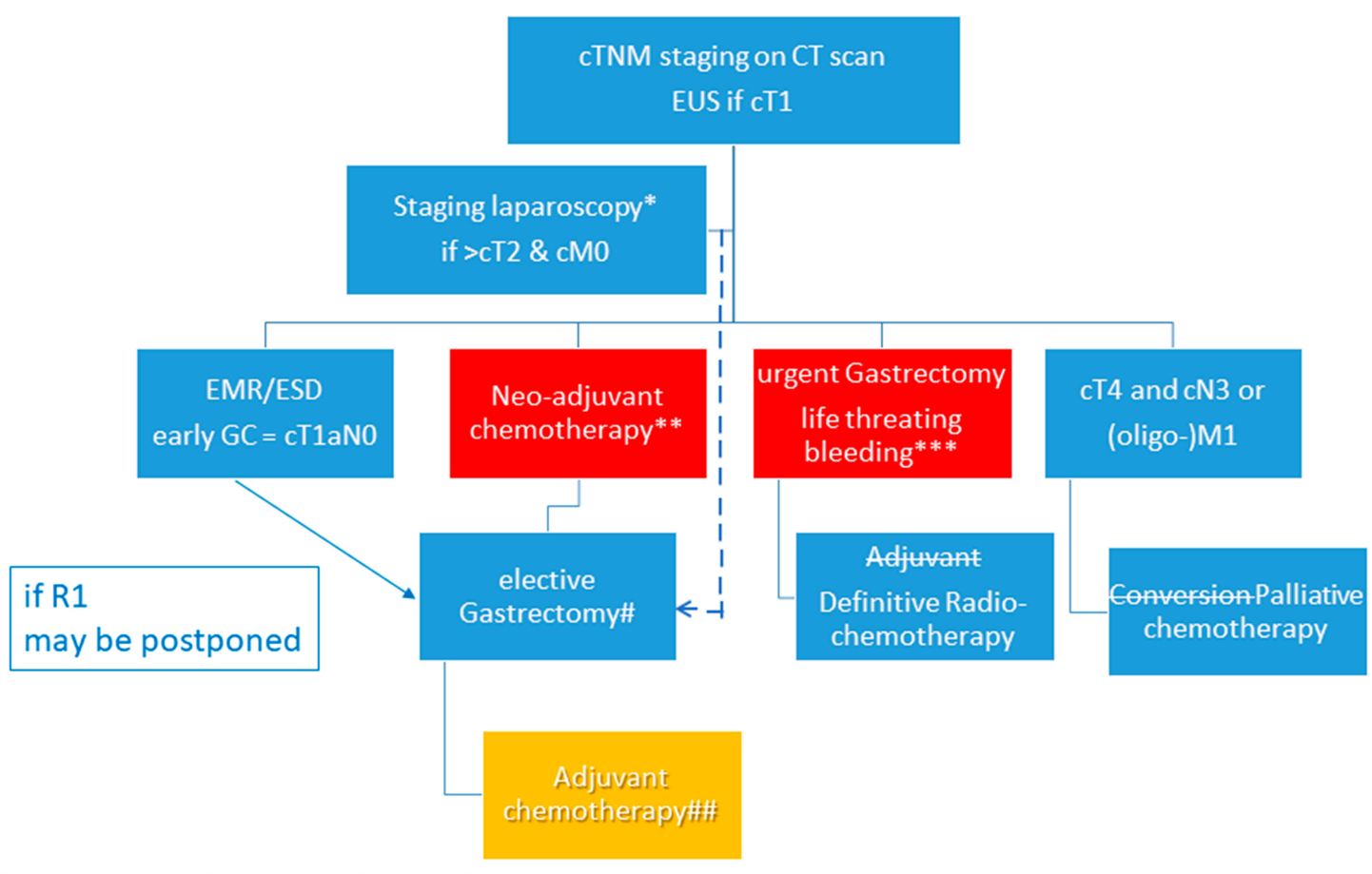

*necessary in diffuse type according to Laurén classification

**except MSI high

***obstruction treated by endoscopic stent

\# if easy resectable with low risk of respiratory complications

\#\# only for $\mathrm{pN}+$

Figure I Treatment algorithm of GC patients during the COVID-19 pandemic with limited health care resources. 
o option: HIPEC for limited peritoneal dissemination (CY1; $\mathrm{PCI}<7 ; \mathrm{P} 1 / 2)$ should be deferred

- adjuvant chemotherapy reserved only for patients with lymph node $\mathrm{pN}$-positive tumors ${ }^{48}$

- for bleeding GC, endoscopic management is the first choice option, followed by radiotherapy, and urgent gastrectomy reserved only for life-threatening hemorrhage

- perforation of GC with peritonitis should be treated minimally-invasive, with peritoneal lavage and drainage, after no improvement with intravenous broadspectrum antibiotic therapy

- for non-resectable or borderline resectable tumors with high risk of complications, primary radical radiochemotherapy should be proposed

- cases with clinical response might be re-evaluated for resectability in the post-pandemic time

o otherwise (no tumor/lymph node regression) this radiotherapy should be regarded as definitive treatment

- all other loco-regionally advanced (cT4 or cN3) and metastatic (M1; including CY1 or P1-3 following staging laparoscopy) cases should be treated with palliative chemotherapy if performance status is good

- conversion therapy (surgical resectability reevaluation) postponed to the post-pandemic era ${ }^{49}$

Emergency presentation of GC is uncommon, usually associated with an advanced stage and lower rates of operability. The necessity to perform an emergency operation within 24 hours is exceedingly rare. However, emergency presentation is a marker of poor long-term outcomes for the equivalent cancer stage in non-metastatic (stages IIII) disease. ${ }^{50}$ Almost all perforated gastric ulcers can be effectively managed by laparotomy and omental patch repair. Initial biopsy and follow-up endoscopy with repeat biopsy is essential to avoid missing of underlying malignancy. ${ }^{51} \mathrm{~A}$ balanced surgical strategy using local laparoscopic repair as the first step of surgery, followed by radical open gastrectomy with lymphadenectomy may be considered. ${ }^{52}$ Perforation of the stomach should raise suspicion of malignancy, particularly in elderly patients. At the time of perforation radical gastrectomy with lymphadenectomy is mostly not advised, either because a diagnosis of GC is not confirmed or because the patient's condition does not allow extended surgery. In this situation, it is suggested to consider a two-stage procedure and direct the primary operation at the treatment of perforation and peritonitis. Tumor staging can be completed when the patient has recovered and a radical operation with curative intent can be planned without compromising long-term prognosis. This staged strategy proposed by German authors at the end of the twentieth century would be of special interest in the pandemic era. ${ }^{53}$ Of note, primary laparoscopic repair and postponed radical gastrectomy several months thereafter, with more precise staging and preferably with regression after induction chemo(radio)therapy, does not preclude long-term survival in a substantial number of patients.

Since metastatic GC is usually incurable and carries a very poor prognosis, palliative chemotherapy remains the standard of care. In the pandemic setting, a palliative surgery for complicated GC, although it can provide a symptomatic benefit, should be performed only for lifethreatening indications. A combination of chemotherapy and/or radiotherapy, after thorough multidisciplinary discussions involving the patient, should be offered as a standard first-line treatment. Elective surgery, especially extended and high-risk, can be postponed until resolution of the epidemic restrictions and considered in selected cases with clinical response.

Since metastatic GC patients are not candidates for curative surgical therapy, endoscopic palliation, effective in treating dysphagia or obstruction, should be considered. If possible, nutritional support should be provided by the oral or enteral route and can be organized at home as a component of palliative care. ${ }^{54}$ Owing to the mortality and morbidity associated with surgery in patients with a short life expectancy, surgical palliation has fallen out of favor when compared with local endoscopic therapies. ${ }^{55}$

Bleeding can cause significant morbidity in patients with GC. Surgery may be necessary for GC patients presenting with severe bleeding that cannot be controlled by less invasive, especially endoscopic interventions alone. Transcatheter arterial embolization is a highly effective treatment for advanced gastric cancer with active bleeding. ${ }^{56}$ It should be considered as an additional treatment, especially when endoscopic or surgical treatment fails or when these approaches are difficult.

Palliative radiotherapy can stop bleeding effectively in patients with GC. The data available on bleeding from upper gastrointestinal cancers are limited. ${ }^{57}$ The radiotherapy appears to be a well-tolerated, effective hemostatic agent that should be considered in all patients with bleeding from GC. Commonly used fractionation schedules use 1, 
5 , or 10 fractions. Short fractionation schedules have been used in patients with deteriorating performance status.

A different state of GC management between Asia and the West was confronted with the COVID-19 outbreak that has become a public health emergency. Based on the number of confirmed cases and deaths due to COVID-19 in June 2020, it is intriguing to speculate that the West is more vulnerable to coronavirus.

Therefore, key elements of GC management should be redefined during the COVID-19 pandemic. We propose a framework to provide optimal care for GC patients in the pandemic scenario at a health-care capability. Patients allocation must be based on multidisciplinary decisionmaking and include all anti-cancer treatment options: surgery, systemic therapy, and radiotherapy. The priority/ CRITCON level ( 2 or above), ${ }^{58,59}$ and staffing dictate adherence to the new algorithm. In this framework, prioritization is a key concept based on the severity of symptoms and the need for urgent (surgical) intervention. We believe that these priorities may improve the delivery of care to all GC patients when pre-pandemic resources are simply not available.

\section{Disclosure}

The authors report no conflicts of interest in this work.

\section{References}

1. Bray F, Ferlay J, Soerjomataram I, Siegel RL, Torre LA, Jemal A. Global cancer statistics 2018: GLOBOCAN estimates of incidence and mortality worldwide for 36 cancers in 185 countries. CA Cancer J Clin. 2018;68(6):394-424. doi:10.3322/caac.21492

2. Arnold M, Abnet CC, Neale RE, et al. Global burden of 5 major types of gastrointestinal cancer. Gastroenterology. 2020. doi:10.1053/j. gastro.2020.02.068

3. Arnold M, Park JY, Camargo MC, Lunet N, Forman D, Soerjomataram I. Is gastric cancer becoming a rare disease? A global assessment of predicted incidence trends to 2035. Gut. 2020;69 (5):823-829. doi:10.1136/gutjnl-2019-320234

4. Chan JSH, Chao ACW, Cheung VCH, et al. Gastrointestinal disease burden and mortality: A public hospital-based study from 2005 to 2014. J Gastroenterol Hepatol. 2019;34(1):124-131. doi:10.1111/ jgh. 14377

5. Available from: https://coronavirus.jhu.edu/map.html. (last updated 17 July 2020). Accessed July 11, 2020.

6. The Global Cancer Observatory (GCO) is an interactive web-based platform presenting global cancer statistics to inform cancer control and research. Available from: http://gco.iarc.fr/. Accessed July 11, 2020.

7. Chen N, Zhou M, Dong X, et al. Epidemiological and clinical characteristics of 99 cases of 2019 novel coronavirus pneumonia in Wuhan, China: a descriptive study. Lancet. 2020;395(10223): 507-513. doi:10.1016/S0140-6736(20)30211-7

8. Wang D, Hu B, Hu C, et al. Clinical characteristics of 138 hospitalized patients with 2019 novel coronavirus-infected pneumonia in Wuhan, China. JAMA. 2020;323(11):1061. doi:10.1001/jama.2020.1585
9. Wang H, Zhang L. Risk of COVID-19 for patients with cancer. Lancet Oncol. 2020;21(4):e181. doi:10.1016/S1470-2045(20) 30149-2

10. COVID-19 Guidelines for Triage of Cancer Surgery Patients; 2020. Available from: https://www.facs.org/covid-19/clinical-guidance/elec tive-case/cancer-surgery. Accessed July 11, 2020.

11. Thiels CA, Hanson KT, Habermann EB, Boughey JC, Grotz TE. Integrated cancer networks improve compliance with national guidelines and outcomes for resectable gastric cancer. Cancer. 2020;126 (6):1283-1294. doi:10.1002/cncr.32660

12. Wang XZ, Zeng ZY, Ye X, Sun J, Zhang ZM, Kang WM. Interpretation of the development of neoadjuvant therapy for gastric cancer based on the vicissitudes of the NCCN guidelines. World J Gastrointest Oncol. 2020;12(1):37-53. doi:10.4251/wjgo.v12.i1.37

13. Smyth EC, Verheij M, Allum W, et al. Gastric cancer: ESMO Clinical Practice Guidelines for diagnosis, treatment and follow-up. Ann Oncol. 2016;27(suppl 5):v38-v49. doi:10.1093/annonc/mdw350

14. Al-Batran SE, Homann N, Pauligk C, et al. Perioperative chemotherapy with fluorouracil plus leucovorin, oxaliplatin, and docetaxel versus fluorouracil or capecitabine plus cisplatin and epirubicin for locally advanced, resectable gastric or gastro-oesophageal junction adenocarcinoma (FLOT4): a randomised, Phase 2/3 trial. Lancet. 2019;393(10184):1948-1957. doi:10.1016/S0140-6736(18)32557-1

15. Sada YH, Smaglo BG, Tan JC, Tran Cao HS, Musher BL, Massarweh NN. Prognostic value of nodal response after preoperative treatment of gastric adenocarcinoma. J Natl Compr Canc Netw. 2019;17(2):161-168. doi:10.6004/jnccn.2018.7093

16. Yoshida K, Yamaguchi K, Okumura N, Tanahashi T, Kodera Y. Is conversion therapy possible in stage IV gastric cancer: the proposal of new biological categories of classification. Gastric Cancer. 2016;19(2):329-338. doi:10.1007/s10120-015-0575-z

17. Russo AE, Strong VE. Gastric cancer etiology and management in asia and the west. Annu Rev Med. 2019;70(1):353-367. doi:10.1146/ annurev-med-081117-043436

18. Muro K, Van Cutsem E, Narita Y, et al. Pan-Asian adapted ESMO Clinical Practice Guidelines for the management of patients with metastatic gastric cancer: a JSMO-ESMO initiative endorsed by CSCO, KSMO, MOS, SSO and TOS. Ann Oncol. 2019;30 (1):19-33. doi:10.1093/annonc/mdy502

19. Rawicz-Pruszynski K, van Sandick JW, Mielko J, Cisel B, Polkowski WP. Current challenges in gastric cancer surgery: european perspective. Surg Oncol. 2018;27(4):650-656. doi:10.1016/j. suronc.2018.08.004

20. Japanese Gastric Cancer. A Japanese gastric cancer treatment guidelines 2018 (5th edition). Gastric Cancer. 2020. doi:10.1007/s10120020-01042-y

21. Bang YJ, Yalcin S, Roth A, et al. Registry of gastric cancer treatment evaluation (REGATE): I baseline disease characteristics. Asia Pac $J$ Clin Oncol. 2014;10(1):38-52. doi:10.1111/ajco.12112

22. Ter-Ovanesov M, Yalcin S, Zalcberg J, et al. Registry of gastric cancer treatment evaluation (REGATE): II treatment practice. Asia Pac J Clin Oncol. 2013;9(4):373-380. doi:10.1111/ajco.12089

23. Berlth F, Yang HK. Minimal-invasive gastrectomy: what the west can learn from the east? Updates Surg. 2018;70(2):181-187. doi:10.1007/ s13304-018-0547-z

24. Kurian AA, Wang L, Grunkemeier G, Bhayani NH, Swanstrom LL. Defining "the elderly" undergoing major gastrointestinal resections: receiver operating characteristic analysis of a large ACS-NSQIP cohort. Ann Surg. 2013;258(3):483-489. doi:10.1097/SLA.0b01 3e3182a196d8

25. Ahmad A, Khan H, Cholankeril G, Katz SC, Somasundar P. The impact of age on nodal metastases and survival in gastric cancer. J Surg Res. 2016;202(2):428-435. doi:10.1016/j.jss.2016.02.043

26. Liu G, Xu M, Gao T, et al. Surgical Compliance and Outcomes in Gastric Cancer: a population-based cohort study. J Cancer. 2019;10 (4):779-788. doi:10.7150/jca.29073 
27. Lee JG, Kim SA, Eun CS, et al. Impact of age on stage-specific mortality in patients with gastric cancer: A long-term prospective cohort study. PLoS One. 2019;14(8):e0220660. doi:10.1371/journal. pone. 0220660

28. Slagter AE, Tudela B, van Amelsfoort RM, et al. Older versus younger adults with gastric cancer receiving perioperative treatment: results from the CRITICS trial. Eur J Cancer. 2020;130:146-154. doi:10.1016/j.ejca.2020.02.008

29. Schendel J, Jost E, Mah M, et al. Gastric cancer management in elderly patients: a population-based study of treatment patterns and outcomes in gastric cancer patients $>/=75$ years from Alberta, Canada. Am J Surg. 2020. doi:10.1016/j.amjsurg.2020.03.006

30. Polkowski W, van Lanschot JJ, Offerhaus GJ. Barrett esophagus and cancer: pathogenesis, carcinogenesis, and diagnostic dilemmas. Histol Histopathol. 1999;14(3):927-944. doi:10.14670/HH-14.927

31. de Martel C, Forman D, Plummer M. Gastric cancer: epidemiology and risk factors. Gastroenterol Clin North Am. 2013;42(2):219-240 doi:10.1016/j.gtc.2013.01.003

32. Colquhoun A, Arnold M, Ferlay J, Goodman KJ, Forman D, Soerjomataram I. Global patterns of cardia and non-cardia gastric cancer incidence in 2012. Gut. 2015;64(12):1881-1888. doi:10.1136/ gutjnl-2014-308915

33. Dudeja V, Habermann EB, Zhong W, et al. Guideline recommended gastric cancer care in the elderly: insights into the applicability of cancer trials to real world. Ann Surg Oncol. 2011;18(1):26-33. doi:10.1245/s10434-010-1215-9

34. Holscher AH, Law S. Esophagogastric junction adenocarcinomas: individualization of resection with special considerations for Siewert type II, and Nishi types EG, E=G and GE cancers. Gastric Cancer. 2020;23(1):3-9. doi:10.1007/s10120-019-01022-X

35. Polkowski WP, Mielko J, Geca K, et al. Proximal gastric resection with posterior esophago-gastrostomy and partial neo-fundoplication in the treatment of advanced upper gastric carcinoma. Dig Surg. 2020;37(2):119-128. doi:10.1159/000497452

36. Ma FH, Hu HT, Tian YT. [Surgical treatment strategy for digestive system malignancies during the outbreak of novel coronavirus pneumonia]. Zhonghua Zhong Liu Za Zhi. 2020;42:E001. Chinese

37. Chen YH, Peng JS. [Treatment strategy for gastrointestinal tumor under the outbreak of novel coronavirus pneumonia in China] Zhonghua Wei Chang Wai Ke Za Zhi. 2020;23(2):I-IV. Chinese. doi:10.3760/cma.j.issn.1671-0274.2020.02.001

38. Tuech JJ, Gangloff A, Di Fiore F, et al. Strategy for the practice of digestive and oncological surgery during the Covid-19 epidemic. J Visc Surg. 2020;157(3):S7-S12. doi:10.1016/j.jviscsurg.2020.03.008

39. Bartlett EK, Roses RE, Kelz RR, Drebin JA, Fraker DL, Karakousis GC. Morbidity and mortality after total gastrectomy for gastric malignancy using the American college of surgeons national surgical quality improvement program database. Surgery. 2014;156 (2):298-304. doi:10.1016/j.surg.2014.03.022

40. Wang WJ, Li R, Guo CA, et al. Systematic assessment of complications after robotic-assisted total versus distal gastrectomy for advanced gastric cancer: A retrospective propensity score-matched study using Clavien-Dindo classification. Int $J$ Surg. 2019;71:140-148. doi:10.1016/j.ijsu.2019.09.029

41. Fujiya K, Irino T, Furukawa K, et al. Safety of prolonged wait time for gastrectomy in clinical stage I gastric cancer. Eur J Surg Oncol. 2019;45(10):1964-1968. doi:10.1016/j.ejso.2019.06.006

42. Oh SY, Lee JH, Lee HJ, et al. Natural history of gastric cancer: observational study of gastric cancer patients not treated during follow-up. Ann Surg Oncol. 2019;26(9):2905-2911. doi:10.1245/ s10434-019-07455-z
43. Bhogal RH, Patel PH, Doran SLF, et al. Approach to upper GastroIntestinal cancer surgery during the COVID-19 pandemic Experience from a UK cancer centre. Eur J Surg Oncol. 2020. doi:10.1016/j.ejso.2020.05.022

44. Pietrantonio F, Morano F, Niger M, et al. Systemic treatment of patients with gastrointestinal cancers during the COVID-19 outbreak: COVID-19-adapted recommendations of the national cancer institute of milan. Clin Colorectal Cancer. 2020. doi:10.1016/j.clcc.2020. 05.004

45. Rawicz-Pruszynski K, Mielko J, Pudlo K, et al. Yield of staging laparoscopy in gastric cancer is influenced by Lauren histologic subtype. J Surg Oncol. 2019;120(7):1148-1153. doi:10.1002/ jso.25711

46. Martin D, Mantziari S, Demartines N, Hubner M, Group ESAS. Defining major surgery: a delphi consensus among european surgical association (ESA) members. World J Surg. 2020;44(7):2211-2219. doi:10.1007/s00268-020-05476-4

47. Baiocchi GL, Giacopuzzi S, Marrelli D, et al. International consensus on a complications list after gastrectomy for cancer. Gastric Cancer. 2019;22(1):172-189. doi:10.1007/s10120-018-0839-5

48. Rawicz-Pruszynski K, Cisel B, Mlak R, et al. The role of the lymph node ratio in advanced gastric cancer after neoadjuvant chemotherapy. Cancers. 2019;11(12):12. doi:10.3390/cancers1112 1914

49. Mielko J, Rawicz-Pruszynski K, Skorzewska M, et al. Conversion Surgery with HIPEC for Peritoneal Oligometastatic Gastric Cancer. Cancers. 2019;11(11):11. doi:10.3390/cancers11111715

50. Vasas P, Wiggins T, Chaudry A, Bryant C, Hughes FS. Emergency presentation of the gastric cancer; prognosis and implications for service planning. World J Emerg Surg. 2012;7(1):31. doi:10.1186/ 1749-7922-7-31

51. Leeman MF, Skouras C, Paterson-Brown S. The management of perforated gastric ulcers. Int $J$ Surg. 2013;11(4):322-324. doi:10.1016/j.ijsu.2013.02.010

52. Tsujimoto H, Hiraki S, Sakamoto N, et al. Outcome after emergency surgery in patients with a free perforation caused by gastric cancer. Exp Ther Med. 2010;1(1):199-203. doi:10.3892/etm 00000032

53. Lehnert T, Buhl K, Dueck M, Hinz U, Herfarth C. Two-stage radical gastrectomy for perforated gastric cancer. Eur J Surg Oncol. 2000;26 (8):780-784. doi:10.1053/ejso.2000.1003

54. Fletcher J, Woodham D, Dera M, Cooper SC. Home parenteral nutrition in patients receiving palliative care: a curriculum-based review. Frontline Gastroenterol. 2019;10(4):421-426. doi:10.1136/ flgastro-2018-101113

55. Halpern AL, McCarter MD. Palliative Management of Gastric and Esophageal Cancer. Surg Clin North Am. 2019;99(3):555-569. doi:10.1016/j.suc.2019.02.007

56. Cho SB, Hur S, Kim HC, et al. Transcatheter arterial embolization for advanced gastric cancer bleeding: A single-center experience with 58 patients. Medicine. 2020;99(15):e19630. doi:10.1097/MD.0000000 000019630

57. Hughes C, Radhakrishna G. Haemostatic radiotherapy for bleeding cancers of the upper gastrointestinal tract. Br J Hosp Med. 2019;80 (10):579-583. doi:10.12968/hmed.2019.80.10.579

58. Available from: https://www.england.nhs.uk/coronavirus/wp-content /uploads/sites/52/2020/04/C0239-Specialty-guide-Essential-Cancersurgery-and-coronavirus-v1-70420.pdf. Accessed July 11, 2020.

59. Available from: http://www.moralbalance.org/covid19/covid19blog/. Accessed July 11, 2020. 


\section{Publish your work in this journal}

Cancer Management and Research is an international, peer-reviewed open access journal focusing on cancer research and the optimal use of preventative and integrated treatment interventions to achieve improved outcomes, enhanced survival and quality of life for the cancer patient.

The manuscript management system is completely online and includes a very quick and fair peer-review system, which is all easy to use. Visit http://www.dovepress.com/testimonials.php to read real quotes from published authors. 